\title{
Caracterización del Esqueleto Apendicular de Saimiri sciureus
}

\author{
Characterization of the Appendicular Skeleton of SaImiri sciureus
}

Julio César Blanco Rodríguez ${ }^{1,2}$, María Antonia Montilla Rodríguez ${ }^{1}$, Jhon Fredy Suárez $^{1}$, Margarita Alvarez Herrera ${ }^{1}$, Rubén Mauricio Cuéllar ${ }^{1}$

\section{Resumen}

Se describen las estructuras óseas de las extremidades anteriores y posteriores del primate Saimiri sciureus. Se trabajó con los cadáveres de cinco animales que habían sido decomisados y mantenidos en observación médica en las instalaciones del Hogar de Paso para Fauna Silvestre, Caquetá, Colombia. Se presenta la descripción detallada de cada hueso, incluyendo las medidas promedio.

Palabras clave: Saimiri sciureus, apendicular, osteología, Amazonia

\section{Abstract}

The bones of the anterior and posterior limbs of the primate Saimiri sciureus are described. Five dead specimens that were seized and kept under medical observation at the «Hogar de Paso para Fauna Silvestre», Caquetá, Colombia were used for the study. Detailed description of each bone is presented, including the average measures.

Key words: Saimiri sciureus, appendicular, osteology, Amazonia

\footnotetext{
${ }^{1}$ Facultad de Medicina Veterinaria y Zootecnia, Universidad de la Amazonia, Florencia, Caquetá, Colombia

${ }^{2}$ E-mail: mvzjulioblanco@gmail.com
}

Recibido: 30 de mayo de 2014

Aceptado para publicación: 28 de diciembre de 2014 


\section{INTRODUCCIÓN}

El mono ardilla (Saimiri sciureus) es un primate neotropical de la familia Cebidae (Subfamilia Cebinae), nativo de países de Sudamérica y el Caribe. Es un animal diurno que vive en grupos grandes, de 25 a 100 individuos. Se alimenta principalmente de insectos y pequeños frutos maduros como higos $\mathrm{y}$ néctar, trepa para alcanzar frutos en la copa de los árboles más altos y pasa gran parte del día recorriendo el bosque en busca de insectos, especialmente orugas y ortópteros grandes (Emmons, 1999).

La longitud cabeza-cuerpo es de 265 a $370 \mathrm{~mm}$. La cola es bastante versátil y la usa como elemento de equilibrio y moderadamente como palanca, sin que sea prensil, y mide entre 360 y $450 \mathrm{~mm}$. La cara está delineada por un antifaz color blanco, a excepción del hocico que es negro. Las orejas y la garganta son blancas y la espalda está teñida de canela amarillento. El vientre es blanco o blanco amarillento y el último tercio de la cola es negruzco o negro (Defler, 2010).

Los trabajos de Sherwod y Parsons (1985) describen el cuerpo de los vertebrados silvestres y hacen un estudio comparativo de su estructura; sin embargo, no presentan un análisis detallado de los órganos, ni cubre varias especies de animales que deben ser analizadas. Asimismo, el libro clásico de anatomía de Sisson y Grossman (Getty, 2000) no contempla información sobre animales silvestres.

En Colombia, son pocas las experiencias en este tema. Varela (2005) describe las características externas de primates de la familia Cebidae y aspectos breves sobre los sistemas y órganos de los sentidos. Por otro lado, Defler (2010) describe aspectos taxonómicos, de comportamiento, distribución geográfica e historia natural de especies de primates neotropicales, incluyendo a $S$. sciureus. Es así que el presente trabajo tuvo por finalidad describir el sistema óseo correspondiente al esqueleto apendicular, como aporte importante para el estudio de la anatomía de mamíferos silvestres.

\section{Materiales y Métodos}

El estudio se llevó a cabo en la granja experimental Santo Domingo de la Universidad de la Amazonia, en la zona noroccidental del departamento de Caquetá, Colombia. La granja se localiza a $7 \mathrm{~km}$ de la ciudad de Florencia, a $1^{\circ} 36^{\prime} 26^{\prime \prime} \mathrm{N}$ y $75^{\circ} 31^{\prime} 58^{\prime \prime} \mathrm{W}$, con una altitud promedio de $280 \mathrm{msnm}$. La región se clasifica, según Holdridge (1978), como bosque húmedo tropical, con una precipitación pluvial media anual de $3835 \mathrm{~mm}$, promedio de humedad relativa entre 79.5 y $88.6 \%$ y temperatura del aire media anual de $26^{\circ} \mathrm{C}$.

Se utilizaron cadáveres de primates machos adultos de la especie Saimiri sciureus. Los animales habían sido decomisados y mantenidos en observación médica en las instalaciones del Hogar de Paso para Fauna Silvestre (HPFS), pero fallecieron a causa de las deficientes condiciones de cautiverio a las que fueron sometidos previo al decomiso.

Los cuerpos que se encontraban en un grado de descomposición apropiado para la investigación fueron exhumados $(\mathrm{n}=5)$, siguiendo las normas de asepsia respectivas para proteger al personal y prevenir posible difusión de patógenos. Los cadáveres se sometieron a una labor de limpieza siguiendo la metodología de Kusmeluk (2014), mediante uso de una escobilla para eliminación de residuos (polvo, tierra y pelos), con posterior proceso de desengrasado mediante inmersión en recipientes metálicos con detergente al calor hasta punto de ebullición, a fin de eliminar los tejidos solubles adheridos. Finalizado el proceso de limpieza, las estructuras se sumergieron en peróxido de hidrógeno durante 48 horas, con exposición posterior al sol para reducir la humedad. 
Cuadro 1. Estructuras óseas que conforman el esqueleto apendicular de Saimiri sciureus

\begin{tabular}{ll}
\hline Miembro torácico & Miembro pelviano \\
\hline Clavícula & Ilion \\
Escápula & Isquion \\
Húmero & Pubis \\
Radio & Fémur \\
Ulna & Rótula \\
& Tibia \\
& Peroné \\
Carpo $(\mathrm{n}=8-9)$ & Tarso $(\mathrm{n}=7)$ \\
Metacarpo $(\mathrm{n}=5)$ & Metatarso $(\mathrm{n}=5)$ \\
Falanges ${ }^{1}$ & Falanges ${ }^{1}$ \\
\hline${ }^{1}$ Tres por cada dedo excepto el primero
\end{tabular}

Una vez culminado el proceso de secado, los componentes óseos fueron separados con bisturí para su medición y descripción anatómica. Solo se utilizaron los huesos del miembro torácico y pelviano (la osamenta del esqueleto axial será utilizada en otro estudio). Para la toma de medidas óseas se utilizó un calibrador manual, también conocido como pie de rey (vernier), el cual presenta una precisión de milésimo de pulgada (Flores, 2010).

La descripción de cada una de las estructuras se desarrolló a partir de la metodología descriptiva utilizada por Getty (2000), y considerando los preceptos teóricos de la Nómina Anatómica de Veterinaria (para la representación anatómica de los animales domésticos). Las mediciones se realizaron en los huesos de cada lado (derecho e izquierdo, respectivamente).

\section{Resultados Y Discusión}

Se identificaron 130 huesos en el esqueleto apendicular de Saimiri sciureus (Cuadro 1), sin considerar los sesamoideos.

\section{Miembro Torácico}

\section{Clavícula}

Es un hueso corto, dispuesto transversalmente entre el manubrio del esternón y la escápula.

\section{Escápula}

Es un hueso aplastado y delgado (Fig. 1A), de forma triangular, coincidente con lo descrito por Frandson (1995). Presenta dos superficies denominadas costal y lateral (ICVGAN 2012), tres bordes y tres ángulos. La superficie lateral se divide en dos fosas desiguales por la espina de la escápula, que se extiende desde el borde dorsal hasta el cuello del hueso. La fosa supraespinosa es más pequeña que la fosa infraespinosa, la cual es ancha y lisa en su parte superior y más estrecha hacia la parte inferior. Cerca del cuello se encuentra el forámen nutricio. La superficie costal se encuentra profundizada parcialmente por la fosa subescapular, siendo más notoria hacia la parte media. Presenta dos o tres crestas oblicuamente ascendentes para la inserción del músculo subescapular.

El borde craneal es convexo y liso en su parte dorsal, y ligeramente cóncavo hacia su parte media. El borde caudal es recto y delgado en su tercio dorsal y grueso ventralmente, sin presentar concavidades visibles. El borde dorsal es ligeramente convexo, con una marcada curvatura hacia el ángulo craneal. Este se encuentra ubicado entre los bordes craneal y dorsal, y es relativamente delgado y ligeramente convexo. El ángulo caudal es medianamente grueso, con tendencia a formar ángulo recto.

El ángulo ventral se une al cuerpo del hueso mediante el cuello de la escápula. Presenta un ensanchamiento en forma triangular hacia la parte ventral llamada cavidad glenoidea. La escápula al unirse con la cabeza del húmero forman la articulación escápulo humeral. Hacia la parte craneoventral se encuentra la apófisis coracoides, que se pro- 
yecta caudalmente con una pronunciada curvatura hacia la parte ventral. Las medidas promedio de las estructuras escapulares se encuentran en el Cuadro 2.

\section{Húmero}

Es un hueso alargado (Fig. 1B), que se extiende proximalmente desde la escápula hasta la articulación húmero-radio-cubital. Distalmente se acopla con el radio y cúbito. Consta de una diáfisis y dos epífisis. Las medidas se encuentran en el Cuadro 3.

La diáfisis muestra $1 \mathrm{~cm}$ menos en relación con la longitud total del hueso. Al igual que en el humano, el extremo proximal es cilíndrico y estrecho, sin presentar retorcimiento aparente, y en el extremo distal se presenta de forma triangular, plana y ancha. La cara externa o lateral es lisa, presentando en el tercio medio una tuberosidad deltoidea, pequeña y en forma de cresta.

La superficie articular de la epífisis proximal es circular convexa y, aproximadamente, dos veces más extensa que la cavidad glenoidea de la escápula con la que se

Cuadro 2. Medidas promedio de estructuras de la escápula de Saimiri sciureus $(\mathrm{n}=5)$

\begin{tabular}{lc}
\hline Escápula & $\begin{array}{c}\text { Promedio } \\
(\mathrm{cm})\end{array}$ \\
\hline Borde dorsal & 1.68 \\
Borde caudal & 2.44 \\
Borde craneal & 1.86 \\
Ancho máximo & 1.74 \\
Fosa supraespinosa $^{1}$ & 0.42 \\
Fosa infraespinosa $^{1}$ & 0.86 \\
Apófisis coracoides & 0.76 \\
Espina & 1.66 \\
Cavidad glenoidea & 0.46 \\
\hline${ }^{1}$ Medido en la parte más ancha &
\end{tabular}

Cuadro 3. Medidas promedio del húmero de Saimiri sciureus $(\mathrm{n}=5$ )

\begin{tabular}{lc}
\hline Húmero & $\begin{array}{c}\text { Promedio } \\
(\mathrm{cm})\end{array}$ \\
\hline Longitud total & $5.8 \pm 0.8$ \\
Diáfisis & \\
Longitud & $4.7 \pm 0.08$ \\
Diámetro & $0.2 \pm 0.08$ \\
Epífisis proximal & \\
Longitud & $0.6 \pm 0.08$ \\
Diámetro & $0.7 \pm 0.09$ \\
Epífisis distal & \\
Longitud & $0.4 \pm 0.08$ \\
Diámetro & $1.1 \pm 0.08$ \\
Foramen $(\mathrm{mm})$ & $1 \times 1$ \\
\hline
\end{tabular}

articula. El tubérculo mayor (tuberosidad lateral) se halla situado cráneo-lateralmente y el tubérculo menor (tuberosidad medial) es menos pronunciado. Están separados por el surco intertubercular (ICVGAN, 2012).

La epífisis distal posee la tróclea, superficie que permite articular el cúbito y el radio. A cada lado de la tróclea se encuentran los cóndilos medio y lateral; y cráneo dorsal a esta, se ubica la fosa radial. Hacia el borde cráneo-medial del cóndilo medio, a una distancia de aproximadamente $5 \mathrm{~mm}$ se encuentra un foramen de $1 \times 1 \mathrm{~mm}$. Caudal a la tróclea, se observan dos crestas denominadas epicóndilo medio y epicóndilo lateral, que presenta lateralmente la cresta epicondiloidea. Entre los epicóndilos se halla la fosa del olécranon, que es profunda y dentro de la cual se proyecta la apófisis ancónea del cúbito.

\section{Radio}

Hueso largo de $5 \mathrm{~cm}$ de longitud, con una diáfisis curvada de $0.1 \mathrm{~cm}$ de diámetro (Fig. 1C). La superficie lateral es lisa, ligeramente convexa casi en su totalidad y redondeada de lado a lado. La superficie medial es 


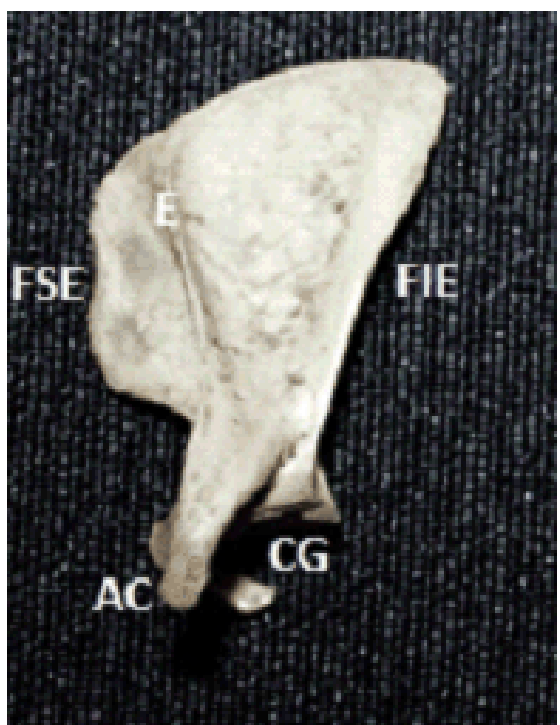

(A)

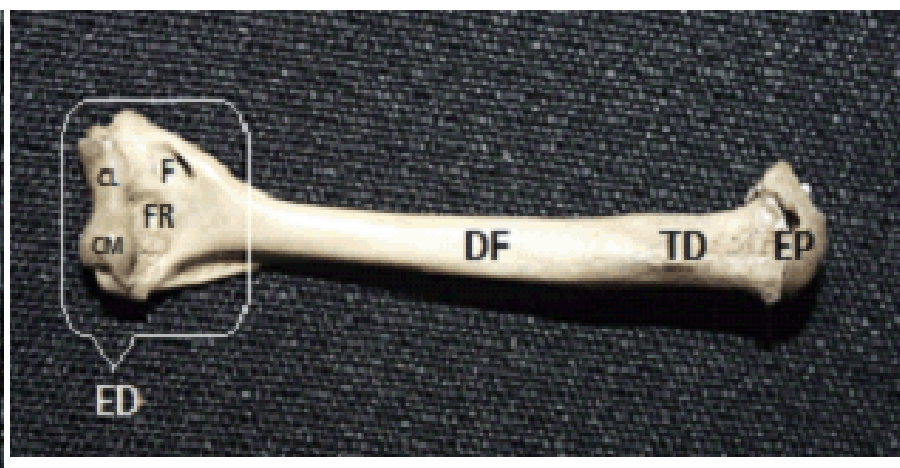

(B)

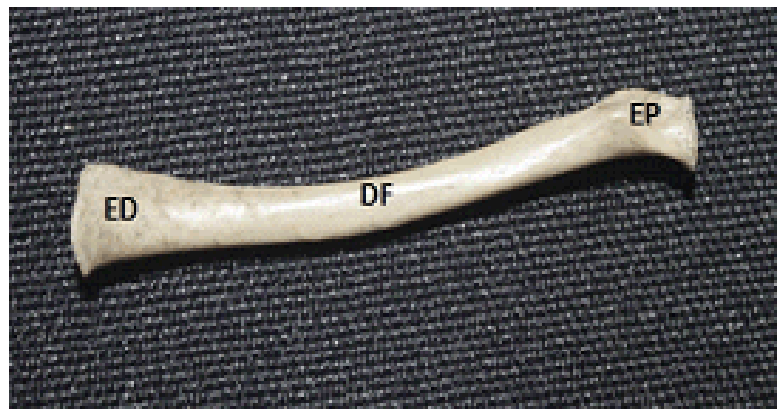

(C)

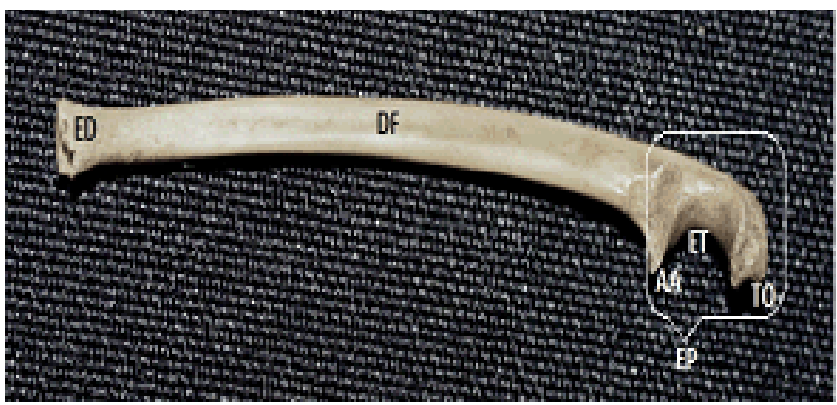

(D)

Figura 1. Huesos del miembro superior de Saimiri sciureus. (A) Escápula. FSE, fosa supraespinosa; FIE, fosa infraespinosa; E, espina; $C G$, cavidad glenoidea; $A C$, apófisis coracoides; (B) Húmero. DF, diáfisis; TD, tuberosidad deltoidea; EP, epifisis proximal; $E D$, epifisis distal; $C M$, cóndilo medio; $C L$, cóndilo lateral; $F$, foramen; FR, fosa radial (C) Radio. DF, diáfisis; ED, epifisis distal; EP, epifisis proximal; (D) Ulna. DF, diáfisis; ED, epifisis distal; EP, epifisis proximal; $A A$, apófisis ancónea; ET, escotadura troclear; TO, tuberosidad olecraniana

cóncava en su longitud y aplanada en dirección transversa. Existe un espacio interóseo entre el cuerpo del cúbito y del radio. Los bordes craneal y caudal son ligeramente redondeados.

La epífisis proximal mide $0.4 \mathrm{~cm}$ de largo; es ancha y aplanada lateralmente. Inme- diatamente distal al borde medial existe una carilla cóncava para la articulación con el cúbito. El foramen nutricio está ubicado $1 \mathrm{~cm}$ por debajo de estas carillas. La epífisis distal, de $0.6 \mathrm{~cm}$ de longitud, es también ancha y aplanada, y presenta una superficie articular carpiana para la fila proximal de los huesos carpianos. 
Ulna

Hueso largo de $5.46 \mathrm{~cm}$ de longitud y $0.12 \mathrm{~cm}$ de diámetro en promedio (Fig. 1D). El cuerpo es ligeramente curvo y presenta dos superficies y dos bordes. Los bordes craneal y caudal son ligeramente lisos y redondeados. La superficie lateral es lisa y aplanada. El olécranon (epífisis proximal), de 0.74 $\pm 0.05 \mathrm{~cm}$ de longitud, se proyecta proximal y caudalmente en el extremo distal del húmero.

La apófisis ancónea es una punta en la parte media craneal de la epífisis proximal del cúbito, de forma más o menos triangular, y sobresale por la incisura troclear (ICVGAN, 2012). Se articula con la fosa olecraniana del húmero. El borde caudal es casi recto, grueso y redondeado. El extremo libre o vértice es una tuberosidad rugosa denominada tuberosidad olecraniana.

\section{La mano} (Fig. 2).

La mano está compuesta por 27 huesos

- El carpo o huesos de la muñeca, según Fleagle (2013), está compuesto de 8 a 9 huesos; sin embargo, en los especímenes evaluados se encontraron ocho huesos. Estos son cortos, pequeños y de forma más o menos cúbica, con dimensiones aparentemente iguales, distribuidos en dos filas: proximal y distal. Ambas filas se conforman de cuatro estructuras, donde la proximal se articula con el radio y la distal con los metacarpos (Fleagle 2013).

- El metacarpo. Son cinco huesos delgados y ligeramente alargados, formados por un cuerpo y dos extremidades. Se numeran de 1 a 5 de adentro hacia afuera, articulándose proximalmente con la fila distal de los carpos y distalmente con las falanges de los dedos. El primero de los huesos es el más corto, el tercero es el más largo. Los metacarpianos consti- tuyen el esqueleto de la región palmar (mano).

- Las falanges. Conjunto de 14 huesos de forma alargada y estructura parecida, pudiéndose distinguir un cuerpo y dos extremidades. Cada dedo tiene tres falanges, a excepción del primer dedo, que solo tiene dos. La falange proximal (primera) se articula con el correspondiente hueso metacarpiano y con la falange media (segunda). La falange media se encuentra situada entre la falange proximal y distal (tercera). La falange distal es libre y termina en el proceso unguicular (uña).

- Los sesamoideos. Huesos de pequeño tamaño que se desarrollan a lo largo de los tendones o en las cápsulas articulares en los puntos en que existen mayores presiones.

\section{Miembro Pelviano}

\section{Hueso coxal}

También llamado hueso de la cadera (Fig. 3A). Es el mayor de los huesos planos, formado por dos estructuras (derecha e izquierda), que se unen firmemente en la parte ventral (sínfisis pelviana). Consta del ilion, isquion y pubis, que se fusionan para formar el acetábulo, fosa profunda y cóncava que se articula con la cabeza del fémur.

\section{Ilion}

Es el mayor y más dorsal de las tres porciones, mide $2.4 \mathrm{~cm}$ de longitud y presenta dos superficies y tres bordes (Fig. 3B). La superficie externa es de forma más o menos cuadrilátera, que se compone del ala, la cual presenta un ancho promedio de $1.1 \mathrm{~cm}$ y un cuerpo de $0.74 \mathrm{~cm}$ que es la parte más estrecha del hueso, evidenciándose la fosa glútea, en la que se cruzan pequeñas líneas glúteas. La superficie pélvica está limitada por la cresta y las líneas arcuatas (poco notorias); la porción interna es rugosa, no homogénea y 


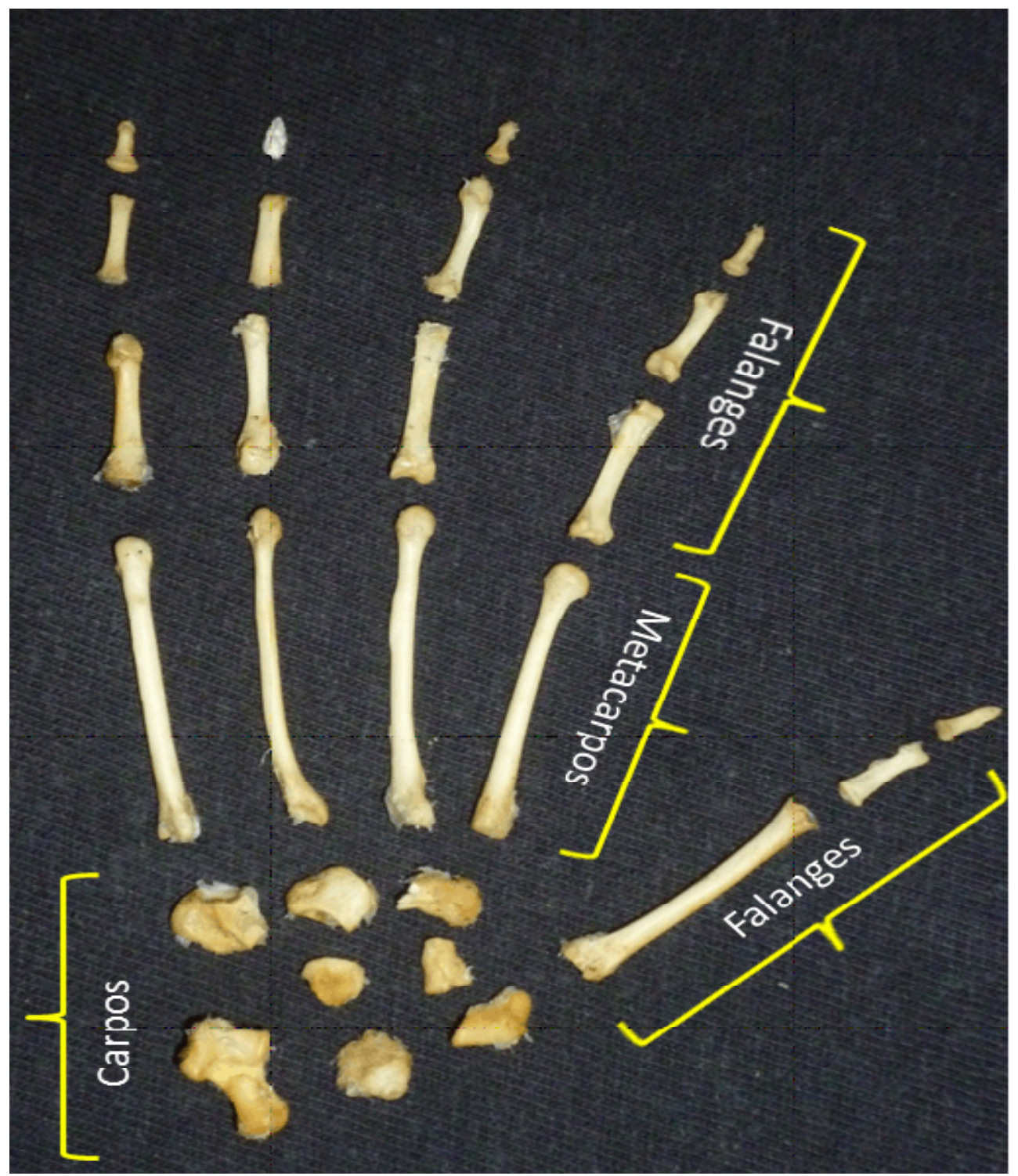

Figura 2. Huesos de la mano de Saimiri sciureu

posee una porción que se articula con el sacro. El borde dorsal es ligeramente recto, ancho y rugoso; su parte media es cóncava y forma la escotadura isquiática mayor, con una longitud de $1.12 \mathrm{~cm}$, que se continúa caudalmente con la espina isquiática $(0.46 \mathrm{~cm}$ de longitud). El borde ventral es cóncavo y en su mayor parte rugoso.

El ángulo medial o tuberosidad sacra es semiredondeado. El ángulo lateral o tuberosidad coxal es rugoso. El acetábulo es la cavidad articular formada por la unión del ilion, pubis e isquion, y posee un diámetro interno promedio de 0.74 centímetros.

\section{Isquion}

El isquion forma la parte caudal de la pared ventral o suelo de la pelvis. Posee una longitud promedio de $1.16 \mathrm{~cm}$. La superficie pélvica es lisa y ligeramente cóncava. La superficie ventral es casi plana, con rugosidades. El borde caudal se une con los 
bordes de su par para formar el arco isquiático. La tuberosidad isquiática es una pequeña masa rugosa que se observa en el ángulo caudolateral. El borde lateral es grueso, redondeado y ligeramente cóncavo, formando la escotadura isquiática menor, que tiene una longitud promedio de $0.56 \mathrm{~cm}$.

\section{Pubis}

Es el más pequeño de los tres y forma la parte craneal del suelo pélvico. Tiene una longitud promedio de $0.78 \mathrm{~cm}$. Está constituido por un cuerpo, dos superficies y tres bordes. La unión de los dos pubis forma la sínfi-

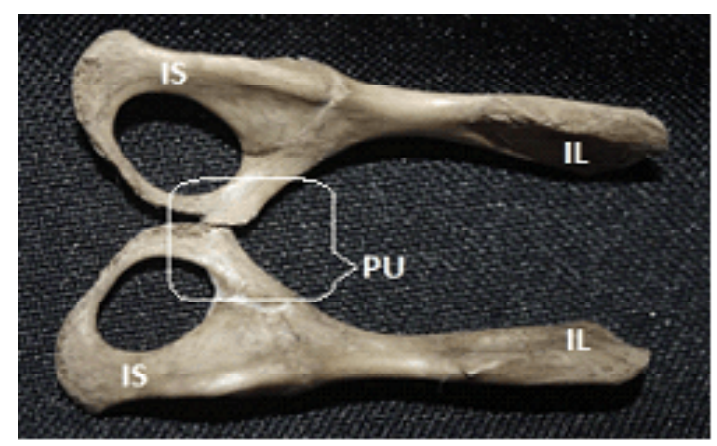

(A)

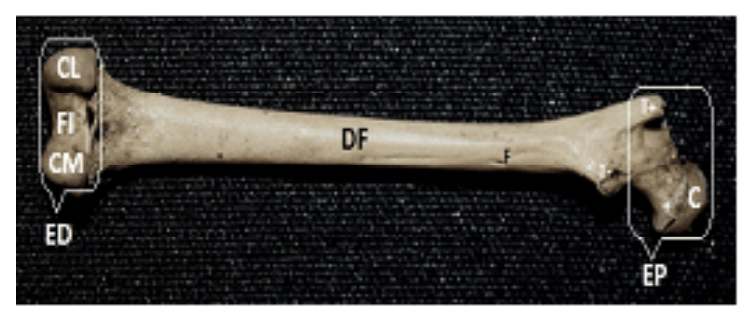

(C)

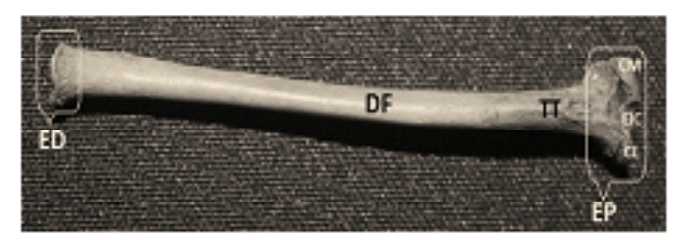

(D)

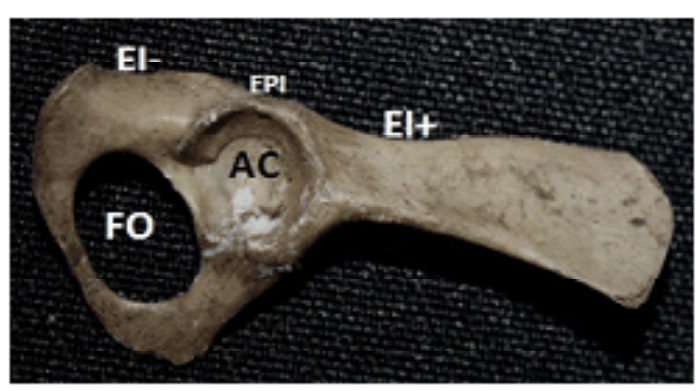

(B)

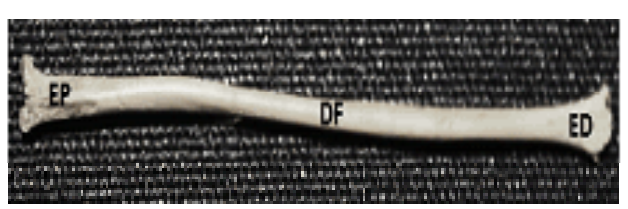

Figura 3. Huesos del miembro inferior de Saimiri sciureus. (A) Coxal: IL, ileon; IS, isquion; $P U$, pubis; (B) Íleon: EI+, escotadura isquiática mayor; EI-, escotadura isquiática menor; EPI, espina isquiática; $A C$, acetábulo; FO, foramen obturador; (C): Fémur: $D F$, diáfisis; EP, epifisis proximal; ED, epifisis distal; $T+$, trocánter mayor; T-, trocánter menor; C, cabeza; CT, cresta trocantérica; FI, fosa intercondiloidea; $C M$, cóndilo medial; $C L$, cóndilo lateral; (D) Tibia: DF, diáfisis; EP, epífisis proximal; ED, epifisis distal; TT, tuberosidad de la tibia; EIC, eminencia intercondiloidea o espina; $C M$, cóndilo medial; $C L$, cóndilo lateral; (E), Peroné: $D F$, diáfisis; EP, epifisis proximal; ED, epifisis distal 
sis púbica, y próxima a esta se evidencia una pequeña prominencia variable, denominada tuberosidad púbica.

\section{Foramen obturador}

Se encuentra entre el pubis y el isquion, sobre el suelo de la pelvis. Presenta un contorno oval, con un diámetro interno de craneal a caudal de $1.08 \mathrm{~cm}$ y de medial a lateral de $0.64 \mathrm{~cm}$.

\section{Fémur}

Es el hueso más largo y fuerte de todos los huesos (Koning y Liebich, 2007). Forma parte del esqueleto del muslo, y posee una longitud promedio de $7.18 \pm 0.15 \mathrm{~cm}$ (Fig. 3C). La variabilidad en el tamaño del fémur observada en el presente estudio podría deberse al sexo, ya que todos eran adultos.

El fémur se articula proximalmente con el acetábulo y distalmente con la tibia y la rótula. Está formado por un cuerpo y dos extremidades. La epífisis proximal presenta una longitud promedio de $0.86 \mathrm{~cm}$. Posee una cabeza casi esférica, con un diámetro promedio de $0.2 \mathrm{~cm}$ y se articula con el acetábulo.

Lateralmente a la cabeza está situado el trocánter mayor y bajo este se ubica la cresta trocantérica. En el borde medial se observa una cresta pequeña, gruesa y rugosa, denominada trocánter menor.

El foramen nutricio se encuentra ubicado aproximadamente a $1 \mathrm{~cm}$ de este trocánter. La diáfisis es cilíndrica, mide $5.88 \mathrm{~cm}$ de largo y tiene un diámetro de $0.2 \mathrm{~cm}$. La epífisis distal mide $1.1 \mathrm{~cm}$ de ancho y $0.44 \mathrm{~cm}$ de largo. En dirección craneal se encuentra la tróclea que se articula con la rótula, que es un hueso sesamoideo de forma esférica, formando la articulación femororrotuliana. Caudalmente se encuentran dos cóndilos, uno medial y otro lateral, que se articulan con la tibia. Los cóndilos están separados por una fosa intercondiloidea, que mide $0.44 \mathrm{~cm}$ de ancho. Presenta, además, los epicóndilos medial y lateral.

\section{Tibia}

Es un hueso largo de $8.38 \mathrm{~cm}$ de longitud (Fig. 3D). Se articula proximalmente con el fémur, distalmente con el tarso y lateralmente con el peroné. Presenta una diáfisis de $6.06 \mathrm{~cm}$ de largo y $0.12 \mathrm{~cm}$ de diámetro. En su superficie caudal, a $1.5 \mathrm{~cm}$ distal a la epífisis proximal se observa el foramen nutricio.

La epífisis proximal es ancha, mide 1.06 $\mathrm{cm}$ de diámetro y $0.32 \mathrm{~cm}$ de largo. Posee dos eminencias articulares, los cóndilos medial y lateral, separados caudalmente por una escotadura. Estas presentan una superficie que se articula con el correspondiente cóndilo del fémur y meniscos. En medio de los dos cóndilos, se observa la eminencia intercondiloidea o espina, formada por una parte medial y una parte lateral. Inmediatamente próximo-craneal, se encuentra ubicada una eminencia grande, la tuberosidad de la tibia. La epífisis distal es mucho más pequeña que la proximal, de $0.54 \mathrm{~cm}$ de ancho, con una superficie articular para el astrágalo, uno de los huesos de la fila proximal del tarso.

\section{Peroné}

Es un hueso largo y fino que se extiende lateralmente desde la epífisis proximal de la tibia hasta la porción lateral del tarso (Fig. 3E). Mide $6.08 \mathrm{~cm}$ de longitud, presenta una diáfisis ligeramente recta de $0.1 \mathrm{~cm}$ de diámetro, formando el límite del espacio interóseo de la pierna. Las epífisis proximal y distal tienen un diámetro aproximado de $0.2 \mathrm{~cm}$, medida que concordó con 4 de los 5 especímenes. Este hueso tiene como principal función la inserción de los músculos y no soporta el peso del cuerpo, como sucede con la tibia (Palastanga et al., 2000). 


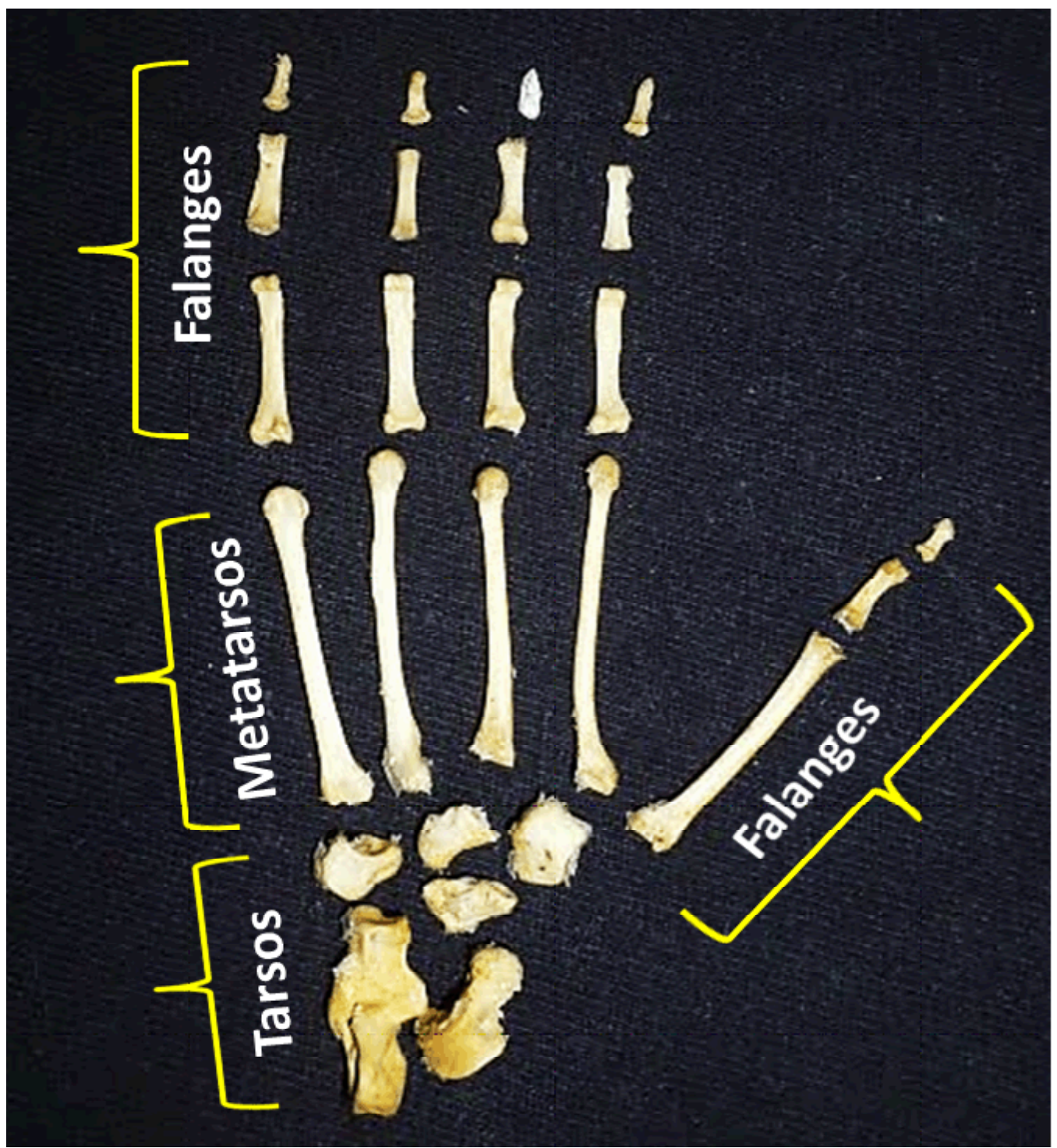

Figura 4. Huesos del pie de Saimiri sciureus

\section{El pie}

El pie del $S$. sciurius está compuesto por 26 huesos (Fig. 4), con una longitud promedio de $6.08 \mathrm{~cm}$.

- El tarso consta de seis huesos, según Fleagle (2013), situados en la parte posterior del pie. En la parte proximal se encuentran dos huesos que hacen parte del tobillo, el astrágalo y el calcáneo. La cabeza del astrágalo se articula con el hueso navicular y este a su vez con tres pequeños huesos cuneiformes, que se articulan con los tres primeros metatarsianos. Al igual que los carpos, estos huesos sirven para amortiguar los impactos.

- Los metatarsos son cinco huesos, de estructura similar a los metacarpianos. Presentan un cuerpo y dos extremidades, se articulan proximalmente con cuatro de los tarsos y distalmente con la primera de las falanges que conforman el dedo. 
- Las falanges y los sesamoideos. Partiendo de las afirmaciones de Fleagle (2013), los dedos de los pies se asemejan a los de la mano en cada uno de los cuatro dígitos laterales que se articulan a partir de una base plana con los metatarsianos; cuya cabeza es redondeada y seguida por tres falanges. El primer dígito es corto y tiene una articulación móvil en su base para agarrar.

Según Fleagle (2013), tanto los pies como las manos de los primates muestran diferencias considerables de una especie a otra, en proporciones relativas a los distintos elementos en asociación con las habilidades del aparato locomotor.

\section{Agradecimiento}

Los autores agradecen al Hogar de Paso de Fauna Silvestre de la Universidad de la Amazonia, coordinado por la Dra. Gloria Elena Estrada Celi, por facilitar el material biológico para el presente estudio. Asimismo, a las autoridades de la Facultad de Ciencias Agropecuarias, por los espacios y materiales suministrados para los estudios que adelanta el semillero de investigación AMERAt, en conjunto con los docentes del área de anatomía, y a CORPOAMAZONIA, por apoyar los procesos de investigación en especies silvestres.

\section{Literatura Citada}

1. Defler TR. 2010. Historia natural de los primates colombianos. Bogotá, Colombia: Univ Nacional de Colombia. 614 p.

2. Emmons L. 1999. Mamíferos de los bosques húmedos de América tropical. Una guía de campo. Santa Cruz de la Sierra, Bolivia: Ed FAN. 298 p.

3. Estrada GE, Castaño DE, Ramírez KJ, Rodríguez JA, Gonzales LA. 2012. Estudio de la eficacia del Paico (Chenopodium ambrosioides) como antihelmíntico, en especímenes silvestres mantenidos en cautiverio en el Hogar de Paso de Fauna Silvestre de la Universidad de la Amazonia. Ces Med Vet Zootec 7:31-36.

4. Fleagle JG 2013. Primate adaptation and evolution. $3^{\text {rd }}$ ed. USA: Academic Press. $442 \mathrm{p}$.

5. Flores CS. 2010. Análisis de Bolton utilizando un método de medición digital y el método de medición manual. Tesis de grado. Lima, Perú: Univ Nacional Mayor de San Marcos. 100 p.

6. Frandson RD. 1995. Anatomía y fisiología de los animales domésticos. $5^{\mathrm{a}} \mathrm{ed}$. Editorial Interamericana. México, D.F. $560 \mathrm{p}$.

7. Getty R. 1982. Sisson y Grossman. Anatomía de los animales domésticos. Pensilvania, EEUU: WB Saunders. 1436 p.

8. Holdridge LR. 1978. Ecología basada en zonas de vida. Serie de libros y materiales educativos $\mathrm{N}^{\circ} 34$. San José, Costa Rica: IICA. 276 p.

9. ICVGAN. 2012. Nómina Anatómica Veterinaria. $5^{\text {a }}$ ed. Asociación Mundial de Anatomistas Veterinarios. [Internet]. Disponible en: http://www.wavaamav.org/Downloads/nav_2012.pdf

10. König H, Liebich HG 2007. Veterinary anatomy of domestic mammals. $3^{\text {rd }}$ ed. Germany: Schattauer. 768 p.

11. Kusmeluk. CE. 2014. Preparación y montaje del esqueleto (2014). Biología animal - Trabajos prácticos. Especialidad Zoología Facultad de Ciencias Exactas Químicas y Naturales, Universidad Nacional de Misiones. [Internet]. Disponible en: http://www.aulavirtualexactas.dyndns.org/

12. Palastanga N, Field D., Soames $R$. 2000. Anatomía y movimiento humano. Estructura y funcionamiento. Barcelona, España: Ed. Paidotribo. 611 p.

13. Varela N. 2005. Consideraciones anatómicas de importancia clínica en los primates neotropicales. Rev Asoc Veterinarios Vida Silvestre 1(1): 15 -27. 